\title{
Path Loss Prediction Model for GSM Fixed Wireless Access
}

\author{
P. Elechi and P.O. Otasowie
}

\begin{abstract}
This research investigated the effect of building materials on GSM signals quality. Measurements were conducted on the GSM network in Nigeria, (MTN, Glo, Airtel and Etisalat) using Radio Frequency Signal Tracker on six different building patterns. The results showed that building with alucoboard wall cladding had the highest signal loss while the sandcrete building/unrusted corrugated iron sheet roof had the least signal loss. Also, a model to predict signal penetration through building walls was developed. It was developed using the principles of Fresnel Refraction Coefficient and the knifeedge diffraction. The total losses from the transmitter to the receiver was modelled as a combination of three different effects; losses due to free-space propagation from transmitter to building; the penetration loss was modelled as a combination of the wall penetration loss and the diffraction loss. The results show that despite the condition of the building walls, movement of people in the environment/room also affected the wireless signal quality as well as the chairs and gadgets in the room. The indoor signal path loss in the rooms increased from when the walls were plastered and continued until when the walls were covered with curtains, both rooms reduced by $4 \mathrm{dBm}$. The mean squared error ranged between $1.6 \mathrm{dBm}$ and $2.1 \mathrm{dBm}$ with a standard deviation between 11.1 and 11.5
\end{abstract}

Index Terms - Building, Path Loss, Signal, GSM network, Penetration

\section{INTRODUCTION}

The need for mobile and wireless communications in this modern society cannot be overemphasized, statistics has shown that in many countries, the use of mobile phone is already higher than the fixed one. They are used everywhere, not only outdoor, but also indoor. In these environments, customers demand a good coverage and quality of service [1] and [2]. Signal Propagation models are widely used extensively in network planning, particularly for conducting feasibility studies and during deployment. These models are also useful for performing interference studies as the deployment proceeds [3]. Though, there exist numerous signal propagation models for open and urban environments, none of these models adequately describe signal penetration in buildings. According to [2], signal losses in buildings contributes to about $31 \%$ of the total GSM signal losses. This is because signal penetration loss is associated with the indoor environment [4] and [5].

The paper is aimed at predicting a path loss model for GSM fixed wireless.

Manuscript published July 31, 2016

P. Elechi is with the Department of Electrical Engineering, Rivers State University of Science and Technology, Port Harcourt, Nigeria. elechi.promise@ust.edu.ng.

P.O. Otasowie is with the Department of Electrical/Electronic Engineering, University of Benin, Benin City, Nigeria.

\section{MATERIALS AND METHOD}

\section{A. Measurement}

Measurements were conducted on five different building made with different materials in Elele, Rivers State, Nigeria. The study was carried out on four GSM service providers (MTN, ETISALAT, GLOBACOM and AIRTEL), to determine their signal penetration through buildings made of different materials using Radio Frequency Signal Tracker (RFST) software. The Radio frequency Signal Tracker installed in a Tecno Tablet was used in carrying out the measurements to determine the signal strength, signal-tonoise ratio (SNR) and the distance from the measurement site to the Base Transceiver Stations (BTS).

The measurements were conducted Mud building with thatched roof (MBTR), Mud building with rusted corrugated iron sheet roof (MBCR), Sandcrete building with unrusted corrugated iron sheet roof (SBUR), Sandcrete building with rusted corrugated iron sheet roof (SBCR), Sandcrete building with unrusted corrugated iron sheet roof/POP ceiling (SBPC), and Building with Alucoboard wall cladding (BAWC). In each of the buildings considered, measurements were conducted for both outdoor and indoor

To compare the indoor signal losses, two buildings made with brick wall and concrete wall with dimensions of $5 \mathrm{~m} \times 3 \mathrm{~m} \times 4 \mathrm{~m}$ and $12.7 \mathrm{~cm}$ wall thickness each were constructed. The doors were made with GMP aluminium and glass material with dimensions $(1.5 \mathrm{~m} \times 0.75 \mathrm{~m})$. Several GSM signal measurements were conducted on each building such as:

1) When the walls were not plastered,

2) When the walls were plastered,

3) When the walls were painted,

4) When the walls were tiled,

5) When curtains were hanged on the doors of the rooms,

6) When chairs and gadgets were placed in the rooms,

7) When 4 persons were randomly moving in the room,

and the differences of the losses recorded using equation (1).

$$
P L_{M}=P L_{n M}-P L_{u W}
$$

where $P L_{M}$ is the path loss due to the wall material, $P L_{n M}$ is the path loss due to the new wall material and $P L_{u W}$ is the path loss of the unplastered wall. The signal path loss of the unplastered brick and concrete walls were measured as $22 \mathrm{dBm}$ and $32 \mathrm{dBm}$ and their difference gives the indoor signal path loss due to the internal condition of the wall. 


\section{B. Model Formulation}

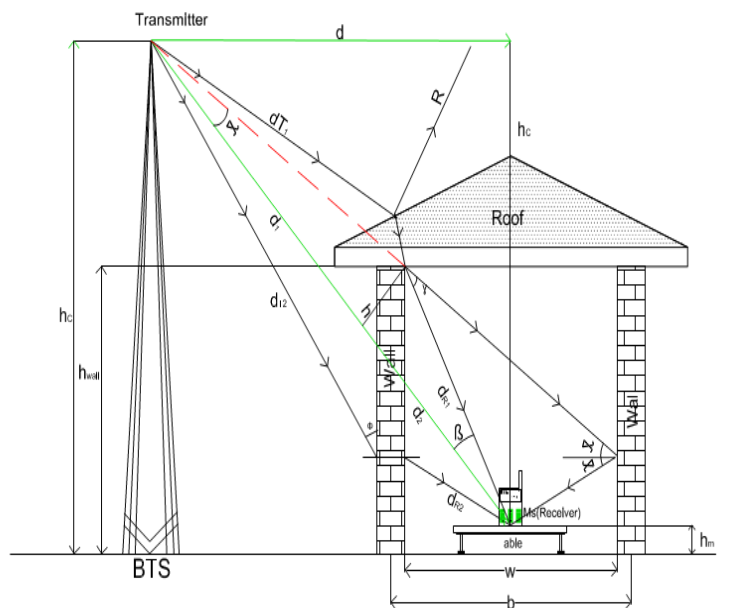

Fig. 1. Complete model of GSM signal penetration into building and parameters used

Fig. 1 is the model used in predicting the amount of GSM signal attenuation through buildings. The model involved the combination of two mechanisms of signal propagation: signal penetration through building wall and penetration through building roof. Though most existing propagation predictions modelled the buildings as being completely opaque to radio signals [6]. The total losses from the transmitter to the receiver was modelled as the combination of different effects; losses due to free-space propagation from transmitter to building, the building wall penetration losses, losses due to trees, human activities, vehicles and other factors. The penetration loss was modelled as the combination of two losses; the loss when the signal is passing through the building wall and diffraction loss due to signal penetration through the roof.

Fig. 1 shows a GSM signal transmission from a BTS to a building. The total losses from the transmitter to the receiver is modelled as [2];

$$
L_{\text {Total }}=L_{f}+L_{p}+L_{d}+G_{a h}+X_{\sigma}+C
$$

where $L_{\text {Total }}$ is the total path loss, $L_{f}$ is the free space loss, $L_{p}$ is the penetration loss, $L_{d}$ is the diffraction loss, $G_{a h}$ is the gain height of the antenna, $X_{\sigma}$ is the log normally distributed variable, $\mathrm{C}$ is the empirical constant or the Raleigh fading.

\section{- Free Space Loss}

The free space loss is given as:

$L_{f}=\frac{4 \pi f d}{c}$

where $\mathrm{f}$ is the carrier frequency, $\mathrm{d}$ is the distance between the transmitter and the building wall and $\mathrm{c}$ is the signal speed.

\section{- Penetration Loss}

The loss of the signal as it passed through the wall is:

$L_{p}=-20 \log _{10} T_{s}$
$T_{s}=\frac{2 \cos \phi}{\cos \phi+\sqrt{\varepsilon_{r}-\sin ^{2} \phi}}$

where $\phi$ is the signal angle of arrival and $\varepsilon_{r}$ is the relative permittivity of the building material.

\section{- Diffraction Loss}

The diffraction loss due to the building obstacle is given as:

$L_{d}=-20 \log _{10}\left(\frac{0.225}{v_{0}}\right)$

$v_{0}=h^{\sqrt{2\left(d_{1}+d_{2}\right)}} / \lambda d_{1} d_{2}$

where $\lambda$ is the signal wavelength, $d_{1}$ is the distance from the transmitter to the diffracting building edge and $d_{2}$ is the distance from the diffracting building edge to the receiver. For obstacles with height higher than the transmitter antenna,

$v_{0}=\sqrt{2}\left[\left(h_{o}-2 h_{R}\right)-\frac{w\left(h_{T}-h_{R}\right)}{d+w}\right] \sqrt{\frac{d \cos ^{2} \phi}{\lambda(d \cos \phi) w}}$

where $h_{T}$ is the receiving antenna height, $h_{T}$ is the transmitting antenna height, $d$ is the distance between the transmitter and the receiver, $\phi$ is the angle of elevation and $h_{0}$ is the medium height of the obstacle.

$h_{0}=\sqrt{r^{2}+\left(\left(K_{e} a_{e}\right)^{2}+2 r K_{e} a_{e} \sin \phi\right)-K_{e} a_{e}+h_{T}}$

where $r$ is the hypotenuse distance between the transmitter and the receiver $a_{e}$ is the radius of the earth and $K_{e}=$ $4 / 3$ (Standard refraction Coefficient).

\section{- Gaussian Random Variable}

The Gaussian random variable was applied to examine the variation of the signals due to human activities and other objects that cause strong signal attenuation.

$C=\frac{L_{p}}{\sigma^{2}} e^{-\left[\frac{L_{p}^{2}}{2 \sigma^{2}}\right]}$

The variations of the measured signal due to human activities and buildings was examined using the $\log$ normally distributed variable.

$X_{\sigma}=\frac{1}{\sigma \sqrt{2 \pi}} e^{-\frac{\left(L_{p}-\overline{L_{p}}\right)^{2}}{2 \sigma^{2}}}$

\section{- Antenna Gain Height}

The antenna gain height was considered in determine the signal gain with respect to antenna height and expressed as [7]:

$G_{T}=20 \log _{10}\left[\frac{h_{R}\left(h_{0}-h_{R}\right)}{h_{T}}\right]$ 
Having computed the received powers and their corresponding distances from the BTS, the least square line method was used to obtain the line of best fit because the best fit curve that has the minimal sum of the deviation $s$ squared for a given set of data.

The least square line approximating the set of points $\left(\mathrm{x}_{1}, \mathrm{y}_{1}\right)$, $\left(\mathrm{x}_{2}, \mathrm{y}_{2}\right)$, $\left(\mathrm{x}_{\mathrm{n}}, \mathrm{y}_{\mathrm{n}}\right)$ has the equation below [8].

$$
y=a x+b
$$

To approximate the set of data $\left(\mathrm{x}_{1}, \mathrm{y}_{1}\right),\left(\mathrm{x}_{2}, \mathrm{y}_{2}\right),\left(\mathrm{x}_{3}, \mathrm{y}_{3}\right),\left(\mathrm{x}_{4}\right.$, $\left.\mathrm{y}_{4}\right), \ldots .\left(\mathrm{x}_{\mathrm{n}}, \mathrm{y}_{\mathrm{n}}\right)$ where $n \geq 2$; such that the sum of squares of the distances to this straight line $y=a x+b$ from the set of points is a minimum.

$$
\begin{gathered}
b=\frac{\left(\sum_{i=1}^{n} y_{i}\right)\left(\sum_{i=1}^{n} x_{i}^{2}\right)-\left(\sum_{i=1}^{n} x_{i}\right)\left(\sum_{i=1}^{n} y_{i} x_{i}\right)}{n\left(\sum_{i=1}^{n} x_{i}^{2}\right)-\left(\sum_{i=1}^{n} x_{i}\right)} \\
a=\frac{n\left(\sum_{i=1}^{n} x_{i} y_{i}\right)-\left(\sum_{i=1}^{n} x_{i}\right)\left(\sum_{i=1}^{n} y_{i}\right)}{n\left(\sum_{i=1}^{n} x_{i}^{2}\right)-\left(\sum_{i=1}^{n} x_{i}\right)}
\end{gathered}
$$

\section{- $\quad$ Mean Squared Error}

The mean squared error of the measured empirical data were obtained using equation 16.

Mean Squared Error $=\left[P_{D}^{2}-M_{D}^{2}\right]$

where $P_{D}$ is the predicted data and $M_{D}$ is the measured data.

\section{RESUlT AND DISCUSSION}

\section{A. Results}

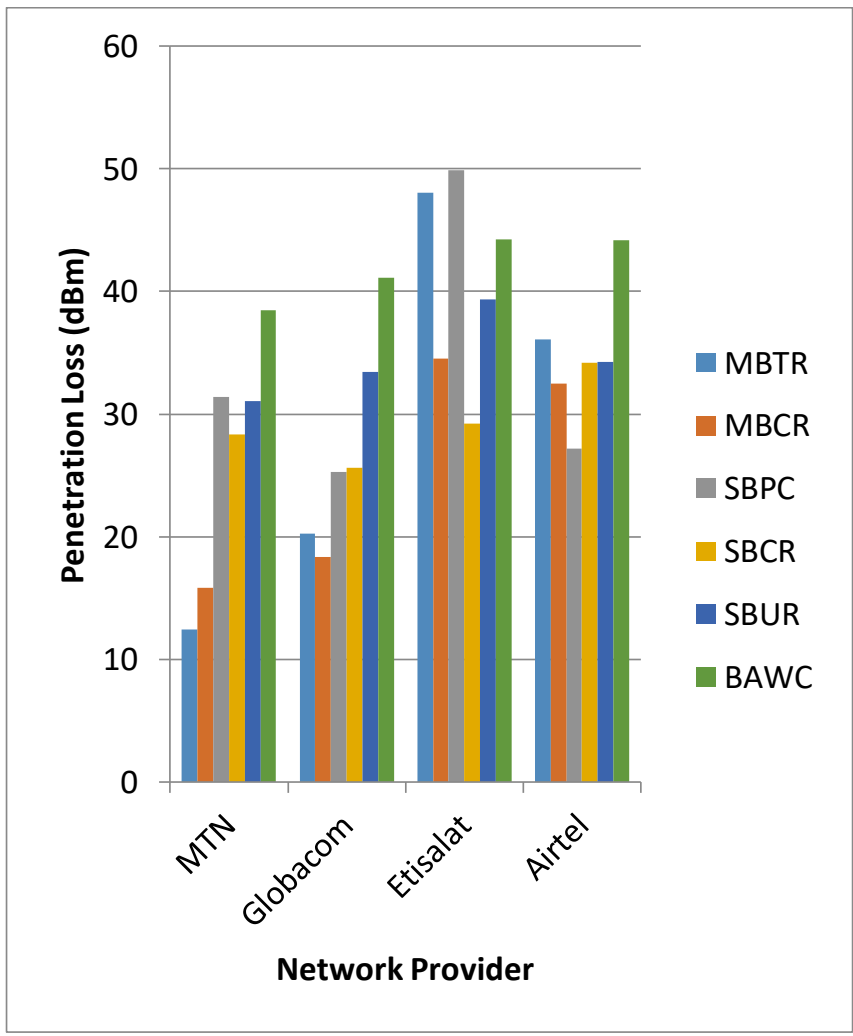

Fig. 2. Average Signal Penetration Loss for Elele

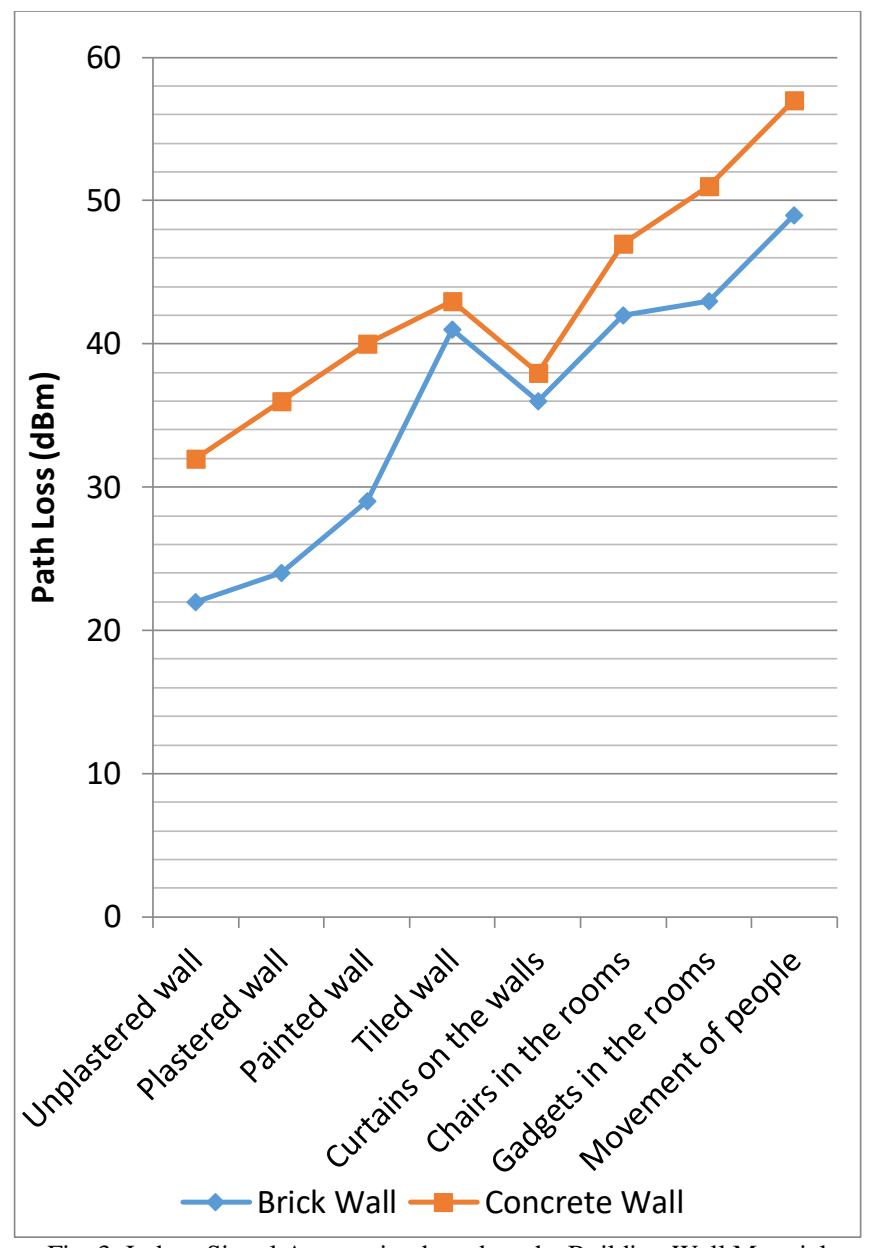

Fig. 3. Indoor Signal Attenuation based on the Building Wall Material

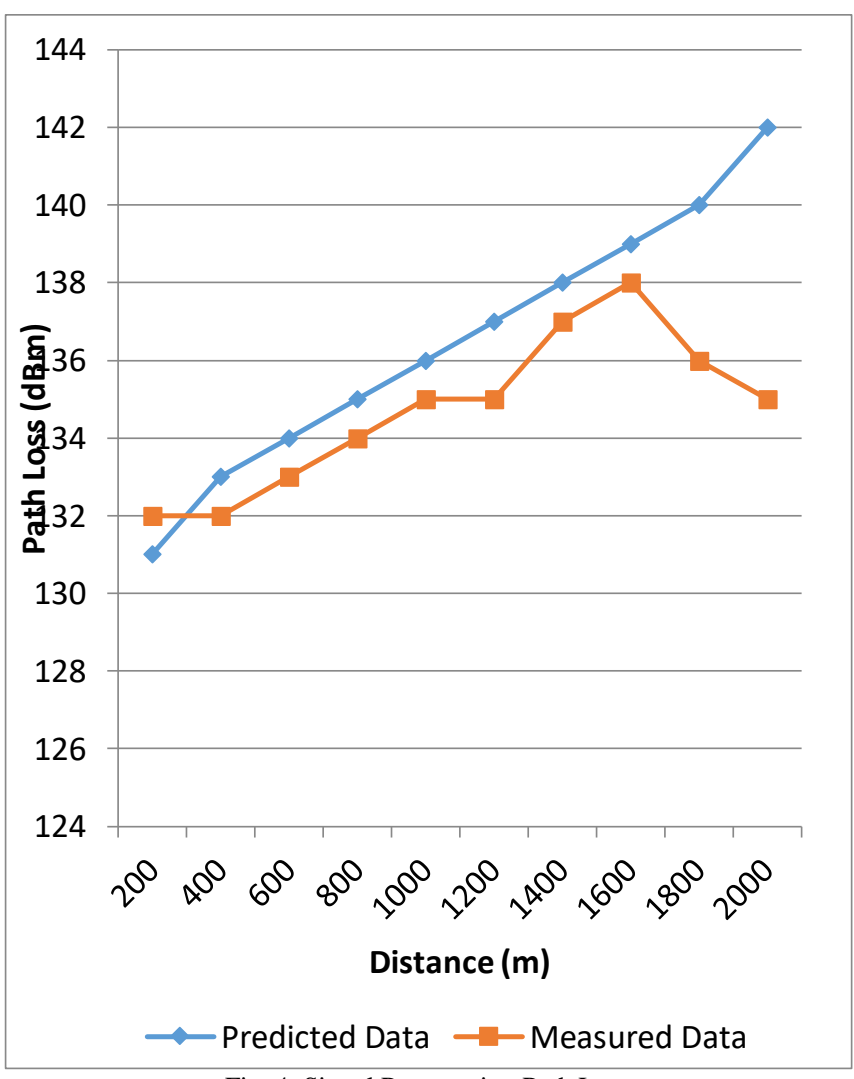

Fig. 4. Signal Propagation Path Loss 


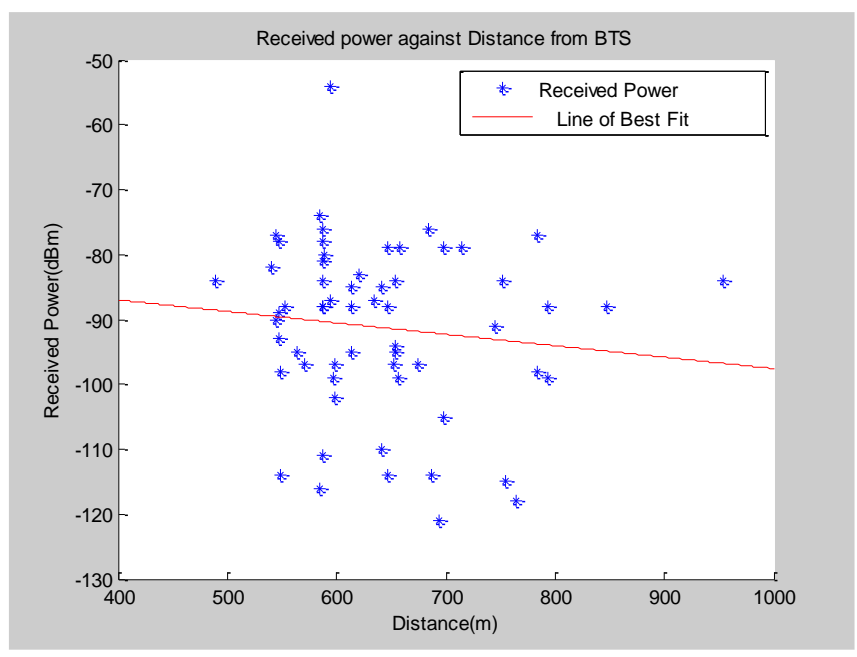

Fig. 5. Scatter points best Fit for Elele Measurements

\section{B. Discussion}

Fig. 5 is the scatter points of the measured signal strength and the line of best fit with equation; $y=-0.017629 x-$ 86.95

This means that for every increase of measurement distance of $1 \mathrm{~m}$, the received signal strength decreases by $0.0017629 \mathrm{dBm}$ and at a minimum distance of 400 meters from the BTS, the received signal strength is $-86.95 \mathrm{dBm}$.

Fig. 2 resents the signal penetration loss for each of the building pattern considered. The results showed that the building with alucoboard wall cladding had the maximum penetration loss while the sandcrete building with rusted corrugated iron sheet roof had the least signal penetration loss.

In Fig. 4, the signal propagation loss is presented and the result shows that the measured data is less than the predicted data with the difference ranging from $1 \mathrm{dBm}$ to $7 \mathrm{dBm}(1 \leq$ $\left.L_{p} \leq 7\right)$. Fig. 3 shows the amount of GSM signal attenuation on the different building wall materials considered. The results show that despite the condition of the building walls, movement of people in the environment/room can also greatly affect the wireless signal quality as well as the chairs and gadgets in the room. The indoor signal path loss in the rooms increased from when the walls were plastered and continued until when the walls were covered with curtains, both rooms reduced by $4 \mathrm{dBm}$. This means that polished walls and movements in an indoor environments affect GSM signal quality.

\section{CONCLUSION}

\section{A. Conclusion}

In this work, a model that can account for the effect of building materials on GSM signal quality was developed. The results showed the effect of building materials on GSM downlink signal power losses. As the mobile equipment (phone) was moved from outside to inside the building, the path loss increased. The penetration loss of the GSM signal was dependent on the building wall/roof material. The results showed that the building with alucoboard wall cladding had the highest signal penetration loss while the sandcrete building/unrusted corrugated iron sheet roof had the lowest signal penetration loss. The mean squared error lied between $1.6 \mathrm{dBm}$ and $2.1 \mathrm{dBm}$ and a standard deviation between 11.1 and 11.5

\section{B. Recommendation}

This research has presented a new model for predicting signal attenuation in an urban and rural environment. Therefore, it is recommend that this study can be extended to other geographical environments such as high climatic environments for effective network planning.

\section{REFERENCES}

[1] P. Elechi and P.O. Otasowie, "Determination of Path Loss Exponent for GSM Wireless Access in Rivers State using Building Penetration Loss," The Mediterranean Journal of Electronics and Communication, vol. 11, no.1, pp. 822-830, 2015.

[2] P. Elechi and P.O. Otasowie, "Analysis of a Developed Building Penetration Path Loss Model for GSM Wireless Access," International Journal of Engineering Research and General Science, vol. 3, no. 6, pp. 898-909, 2015

[3] V.S. Abhayawardhana, I.J. Wassell, D. Crosby, M.P. Sellars, and M.G. Brown, "Comparison of Empirical Propagation Path Loss Models for Fixed Wireless Access Systems," IEEE, pp 213-217, 2003

[4] A.M.D. Turkmani and A.F. Toledo, "Modelling of Radio Transmissions into and within Multistorey Buildings at 900, 1800 and 2300MHz," IEE proc, 1993, pp. 1462-1467, vol. 40 no. 6.

[5] P. Elechi and P.O. Otasowie, "Determination of GSM Signal Penetration Loss in Some Selected Buildings in Rivers State, Nigeria," Nigeria Journal of Technology, vol. 34, no. 3, pp. 609-615, 2015

[6] N.V. Mejuto, "Penetration and Transmission of UHF Radio Waves into/through Buildings-a Literature Review", Graduation Report, Eindhoven University of Technology, 1999.

[7] C.R. Josiane, G.C.F. Simone, S.G. Herminio, and P.S.C. Gervasio, "Path Loss Model for Densely Arboreous Cities in Amazon," in Proc. International Microwave \& Optoelectronics Conference, 2009, pp. 13 .

[8] S.C. Gupta, Fundamentals of Statistics, 6th ed, PVT. Ltd, Himalaya Publishing House, 2011, pp. 8-10.

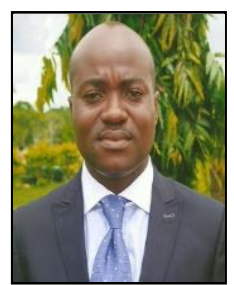

P. Elechi received his B.Eng and M.Eng degrees in Electrical/Electronic Engineering from University of Port Harcourt, Choba, Rivers State, Nigeria in 2006 and 2011 respectively. He is currently undergoing his $\mathrm{Ph} . \mathrm{D}$ study in Electrical/Electronic Engineering (Electronic and Telecommunication), University of Benin. $\mathrm{He}$ is a Corporate Member of the Nigerian Society of Engineers (NSE) and the Nigerian Institution of Electrical/Electronic Engineers (NIEEE). He is also a Registered practicing Engineer with the Council for the Regulation of Engineering in Nigeria (COREN). His research interests are on Radio Propagation for Mobile communications, GSM Technology, Microwave Signal Propagation, Signal Analysis, NanoTechnology and ICT. He is currently a Lecturer in the Department of Electrical and Computer Engineering, Rivers State University of Science and Technology, Port Harcourt, Nigeria.

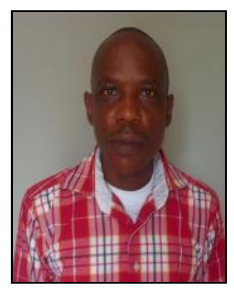

P.O. Otasowie obtained a Ph.D in Electronic and Telecommunication Engineering from the University of Benin, Benin City Nigeria in 2008. He is a corporate member of the Nigerian Society of Engineers, and also registered with the Council for the Regulation of Engineering in Nigeria (COREN). $\mathrm{He}$ is also registered with the IEEE USA. He is currently a Professor in the Department of Electrical and Electronic Engineering, University of Benin, Benin city Nigeria. His research interests are in the area of Mobile Communication, Microwave Signal Propagation, Optical Fibre Communication Systems, and Energy Efficiency in homes and Base Stations among others. 